Early-life circumstances and their effect over the life course*

Florencia Torche

Stanford University

* Florencia Torche, Department of Stanford University, 450 Serra Mall Building 120, Stanford CA 94305, email: torche@stanford.edu. 


\title{
Early-life circumstances and their effect over the life course
}

\begin{abstract}
The idea that early-life circumstances shape people's health, development, and wellbeing over the life course has gained renewed centrality in the last two decades. This renewed interest has been informed by new approaches that emphasize sensitive and critical periods during the first years of life, offer an understanding of human development as a hierarchical and cross-fertilizing process, suggest plausible mechanisms for the persistent effect of early exposures, and explore heterogeneity in effects based on environmental and biological factors. The articles included in this special issue of Population Research and Policy Review advance the field of early-life circumstances in several important dimensions. They examine the determinants and effects of noxious exposures at different developmental stages -ranging from the prenatal period to adolescence- in a variety of national settings. They offer an understanding of early-life circumstances that moves from discrete outcomes to a dynamic life-course approach, and consider diverse sources of heterogeneity in the effects of early exposures.
\end{abstract}




\section{Early-life circumstances and their effect over the life course}

The idea that early life circumstances shape people's health, behavior, and wellbeing over the entire life course is not new. It has been present for centuries, permeating conventional wisdom, art, and literature. For example, in 1667, English poet John Milton put it succinctly in his famous poem Paradise Lost: "The childhood shows the man, As the morning shows the day." (144a, lines 220-21). By the early 20th century, this intuition had already found empirical support in population-level analyses - as when researchers concluded that mortality patterns of cohorts born in the late 19th and early 20th century in England and Sweden behaved "as if the expectation of life was determined by the conditions which existed during the child's early years" (Kermack, McKendrick, and McKinlay 1934)- and had become a central component of public health in many European countries, shaping early reforms to improve the health of mothers and children (Kuh and Smith 1993).

Over the course of the 20th century, however, public health's concern with the early period of life declined as the leading causes of death transitioned from infectious diseases to ischemic heart disease and cancer, and epidemiological models focused on proximate behavioral determinants of health, such as smoking, diet, and exercise (Ben-Shlomo and Kuh 2002; Lynch and Smith 2005). But the turn of the $21_{\text {st }}$ century has brought a renewed emphasis on the initial years of life, driven by a multidisciplinary approach that shows strong effects of early-life circumstances on health, cognition, and attainment, and elucidates biological and social mechanisms for these effects. This renewed concern emerges from two basic tenets: The early period of life -starting at conception- is highly vulnerable to the environment, and it is highly consequential for individual outcomes over the entire life course, and even across generations. As summarized by an interdisciplinary review of the literature published in 2000, "virtually 
every aspect of early human development, from the brain's evolving circuitry to the child's capacity for empathy, is affected by the environments and experiences that are encountered in a cumulative fashion, beginning early in the prenatal period and extending throughout the early childhood years" (Shonkoff and Phillips 2000:6).

Several disciplinary traditions have contributed to this renewed attention to the early stage of life. In the 1970s, pioneering empirical work by physician Anders Forsdahl showed an association between infant mortality rates of cohorts born early in the 20 th century and metabolic disease and arteriosclerotic heart disease mortality in middle age in Norway $(1977,1978)$. Forsdahl hypothesized that this effect emerged from some permanent biological damage caused by nutritional deprivation in-utero, combined with high fat consumption during adulthood in the context of newfound affluence. A similar finding was obtained in Britain, where epidemiologist David Barker observed a strong association between a cohort's birth weight and the risk of coronary disease in late adulthood (Barker and Osmond 1986). These observations, further replicated in many national contexts (Godfrey and Barker 2001), gave rise to the "fetal programming hypothesis." This hypothesis suggests that developments that enable the fetus to adapt to an adverse uterine environment characterized, for example, by nutritional deprivation, may result in permanent programming of developmental patterns, leading to illness and early death due to coronary and metabolic disease (Barker 1997, 1998). The effect of fetal programming usually remains latent for years or even decades, expressing itself in late adulthood irrespective of intervening experience.

Complementarily, research in neurobiology and developmental psychology offered a new conceptual apparatus to explain why exposures at early stages of development have long-term, and even permanent, consequences for individuals. This approach is based on the notions of 
sensitive and critical developmental periods. Sensitive periods are limited developmental stages in which the effect of the environment on a certain capability is stronger (Knudsen 2004). Critical periods are particularly brief and discrete sensitive periods in which the environment may have irreversible effects on a certain capability, regardless of subsequent experience, providing "windows of opportunity" for essential developmental processes (Brown 2005).

The notions of sensitive and critical periods originated in embryology and extended to a wide array of developments of the central nervous system and the brain during early life, with consequences for health, cognition, and behavior (Rice and Barone 2000). Such sensitive and critical periods in brain development were found to "emerge in the prenatal period, reach a peak in the first few years of life, and continue at a declining rate throughout childhood and adolescence" (Hertzman and Boyce 2010:335). A classic example of a critical developmental period is the acquisition of a second language (Hartshorne, Tenenbaum, and Pinker 2018; Lenneberg 1967). If individuals do not learn a second language during a "window of opportunity" that closes in adolescence, full linguistic mastery will never be achieved.

Even though an "opened and closed" view of the effects of experience on development might be an oversimplification when applied to complex outcomes that depend on multiple influences (Bateson 1979; Lynch and Smith 2005; Rutter 1989), there is consensus that early life is highly susceptible to the environment and ripe with developmental opportunity given the high plasticity of neurological and other systems (Gluckman et al. 2008). Environmental influences during the first years of life have persistent consequences at least partly due to epigenetic mechanisms, whereby environmental factors alter gene expression and activity in ways that are persistent and even heritable but which do not involve change in DNA sequence, such as DNA methylation and histone modification. Epigenetic transformations result in the literal embedding 
of early-life experience in the body (Hertzman and Boyce 2010). For example, prenatal exposure to stress appears to induce low birth weight because of the gene expression of so-called stress hormones in the placenta (Hobel 2004; McLean et al. 1995).

The notion of sensitive and critical periods emphasizes that early experience, as opposed to merely prior experience, has lasting impact on individual development and wellbeing. However, the relevance of the early period of life is not exhausted by the fact that sensitive and critical periods are more prevalent during the first years of life. Rather, the relevance of early life is also predicated on a dynamic and hierarchical understanding of human development characterized by what economist James Heckman and collaborators have called self-productivity and dynamic complementarity (e.g. Cunha and Heckman 2007).

Self-productivity refers to the idea that capabilities produced at one stage of development augment the skills attained at later stages, and that capabilities are self-reinforcing and crossfertilizing. For example, good health fosters learning, which in turn may promote emotional security. Dynamic complementarity means that capabilities acquired at one stage of the life course raise the productivity of investment at subsequent stages: for example, mastering basic math concepts makes learning more complex concepts easier.

A life-course approach adds to this understanding of human development an emphasis on the ways various social and biological factors exert their influence over the life course independently, cumulatively, and interactively, linking early-life exposures with developments throughout the life course and shaping trajectories of health, wellbeing and attainment (BenShlomo and Kuh 2002; Hertzman and Boyce 2010; Lynch and Smith 2005; Rutter 1989).

The renewed focus on early circumstances and their effects over the life course has obvious implications for inequality and its persistence across generations. First, noxious 
environmental exposures during early life are patterned by socioeconomic advantage, racial and ethnic privilege, and country, region, and neighborhood of residence. Disadvantaged and minority children living in poor areas are much more likely to be exposed to a wide range of environmental insults such as violence (Harrell et al. 2014), lead poisoning (Muller, Sampson, and Winter 2018), and pollution (Bell and Ebisu 2012), starting at birth. Even if the proximate mechanisms through which these insults affect children's bodies and brains are biological, their fundamental cause is to be found in unequal social structures, usually maintained by institutional mechanisms (Muller et al. 2018).

In addition to stratified exposures in the first years of life, it is also possible that the sensitivity to environmental insults depends on socioeconomic advantage, such that the same exposure has a more detrimental effect among poor children (Torche 2018; Wodtke, Elwert, and Harding 2016). Several theoretical approaches support this hypothesis, including allostatic load (Evans, Li, and Whipple 2013; McEwen and McEwen 2017; McEwen and Stellar 1993), weathering (Geronimus 1992), and cumulative risk factors (Evans et al. 2013; King, Morenoff, and House 2011). These approaches suggest that the "wear and tear" emerging from economic hardship and social exclusion may act as a predisposing factor for the influence of a novel insult; that is, a novel insult will cause more damage to an individual already debilitated by persistent sources of disadvantage. Third, behavioral mechanisms could also contribute to biological and psychological ones: More advantaged families are more likely to identify and mobilize resources to compensate for their children's early disadvantages if they occur (Torche 2018).

To the extent that stratified exposure to environmental insults in early life is compounded by stratified sensitivity and stratified compensatory responses, these processes could result in disadvantage being "locked in" very early in the life course. Unfortunately, because they occur 
so early, the effect of toxic exposures during the first years of life remains invisible unless researchers explicitly look for it, and it is easily, and mistakenly, attributable to genetic or other innate attributes rather than to avoidable environmental factors (Torche 2015, 2018).

Even if there is good theoretical reason to be concerned about early-life circumstances, capturing the effect of specific exposures is a difficult task, posing both methodological challenges and exacting data requirements. A critical methodological challenge is unobserved selectivity. Noxious environmental exposures are usually correlated with unobserved characteristics of the people who experience them as well as with other sources of disadvantage, challenging researchers' ability to disentangle the effect of any single specific factor.

For example, there is a well-established correlation between early-life poverty and decreased cognitive performance (Farah et al. 2006; Johnson, Riis, and Noble 2016). This finding is informative but it raises a critical question: What is it about poverty that hurts children? Is it nutrient deficiencies, financial instability, exposure to pollution, limited cognitive stimulation, exposure to stressors, or other factors? To what extent are differences accounted for by the fact that people who are poor may have different genetic endowments and so would have lower cognitive ability regardless of their poverty status? Disentangling the effects of specific early-life factors associated with poverty is critical to advance science and to craft effective policy interventions.

Researchers have made strides in disentangling these factors by using strategies such as careful multivariate analysis, natural experiments, and neuro-imaging technology. In the case of the association between early-life poverty and cognitive performance, for instance, researchers relying on neuro-imaging have shown that the association between poverty and brain structure and function emerges very early in childhood and is largely independent of genetic variation 
(Hair et al. 2015; Noble et al. 2015). By relying on natural experiments, scholars have isolated several mechanisms that connect poverty with cognitive performance, including exposure to lead (Aizer et al. 2017), acute stress (Torche 2018), local violence (Caudillo and Torche 2014; Sharkey 2010) and social policies such as economic assistance for disadvantaged parents and infants (Hoynes, Miller, and Simon 2015; Hoynes, Page, and Stevens 2011).

An additional challenge in the study of early-life exposures concerns data availability. The seminal analyses of the consequences of early-life conditions in the 1970s and 1980s relied on administrative data aggregated at the local level, for example connecting infant mortality at the county level to adult mortality decades later (e.g. Barker and Osmond 1986; Forsdahl 1977). Recent research relies on similar data disaggregated at the individual level (birth and death records, for example), panel surveys following cohorts starting at birth, and, less frequently, on merged datasets, such as the combination of birth and educational records, or administrative and survey data. These hybrid datasets open new possibilities for following children from conception into adulthood at relatively low cost and high population representativity.

The enormous progress in the understanding of the effect of early-life exposures over the last two decades has led to a new consensus that "a child who falls behind may never catch up" (Heckman 2006:1900). While this claim might be overstated, it invites a research agenda that advances the field by posing questions such as: What are the mechanisms for the effects of earlylife circumstances on the health and wellbeing of populations? How do early exposures shape children's early life-course trajectories as they transition into adolescence and early adulthood? How do these trajectories vary across contexts defined by socioeconomic advantage, genetic makeup, immigration status, gender, and national context, among other sources of heterogeneity and vulnerability? 
This special issue of Population Research and Policy Review addresses these questions. The five contributions included in the issue examine early-life circumstances at different developmental stages - ranging from the prenatal period to adolescence- in a variety of national settings. The contributions are firmly grounded in the new understanding of early life development as a hierarchical process marked by critical developmental windows, and extend this understanding by moving from discrete outcomes to a dynamic life-course approach. This allows the authors to bring issues of unobserved selectivity to the fore, and consider heterogeneity in effects of exposures.

The article Detecting the Effects of Early-Life Exposures: Why Fecundity Matters by Nobles and Hamoudi engages the growing literature on the effect of prenatal exposures on individual outcomes. Nobles and Hamoudi address a critical but neglected issue: survival bias. Starting with the fundamental intuition that prenatal exposures that shape post-birth outcomes could also shape the probability of fetal survival, the authors reason that the individuals observed as exposed to a prenatal shock could in fact be a selected sample of survivors, and thus any outcomes we observe could be shaped by survival bias.

As a poignant example, the authors offer the case of lead-contaminated water in Flint, Michigan. A report by the Michigan Department of Health and Human Services (2017) found virtually no impact of lead-poisoned water on birth outcomes in Flint. However, research has shown that exposure to contaminated water increased fetal deaths by as much as $58 \%$ (Grossman and Slusky 2017). The lack of an observed effect on birth outcomes could, then, be at least partly due to the positive selectivity of pregnancies that survived and became live births in spite of lead poisoning. In order to evaluate the magnitude of survival bias in actual empirical settings, the authors use simulation analysis with realistic parameters, and show that underestimation of the 
detrimental effect of prenatal exposure among survivors could reach $50 \%$ or more, a magnitude that could be as large as the widely appreciated issue of confounding bias.

Nobles and Hamoudi's contribution elegantly shows that selective survivorship in utero is a potentially critical source of bias in any study of the effects of prenatal exposures over the life course. While most researchers would agree with this theoretical possibility, few address it, leaving open the possibility of bias contamination in everything we know about prenatal exposures. Finally, the authors offer suggestions to applied researchers in order to evaluate the plausibility of prenatal survival bias: In addition to assessing a decline in the birth rate and the secondary sex ratio among the exposed population, researchers should examine the variance in the outcome of interest. A substantial reduction of variance would suggest truncation due to fetal loss, and raise a red flag that survival bias is present.

The contribution Birth Weight and Development: Bias or Heterogeneity by Polygenic Risk Factors? by Conley, Sotoudeh and Laidley examines the influence of birth weight on several outcomes during midlife, including body mass index (BMI), height, fluid intelligence test, smoking, and depression/anxiety. Birth weight is shaped by both genetic factors and environmental circumstances in utero. Purging birth weight from the influence of unobserved genetic factors in order to examine the influence of prenatal environmental circumstances is, however, challenging. A common way to do so compares the outcomes of individuals who share their genetic background -- such as siblings and, when possible, twins -- by means of fixed effects models. The use of monozygotic twins provides a particularly strong design because these twins share all their genes, thus any difference in birth weight could be safely attributed to environmental factors. Relying on twin comparisons, several studies have shown that birth weight is consequential for later outcomes net of genetic factors (Black, Devereux, and Salvanes 
2007; Oreopoulos et al. 2012; Torche and Echevarría 2011). Comparing twins is not an infallible strategy, however (Torche and Conley 2015). First, it is possible that variation in birthweight between twins could still be due to factors other than the uterine environment, such as differences in chorionic or placental architecture. Second, by definition twins have the same gestational age so the only source of variation in birth weight emerges from intrauterine fetal growth. As a result, nothing could be inferred from twin studies about differences in birth weight driven by gestational age, which is the main cause of low birthweight in advanced industrial countries.

Conley and colleagues offer an alternative strategy to capture the effect of birth weight net of genetic confounding: they directly control for genetic makeup of individuals by means of polygenic scores predicting each of the outcomes of interest. Polygenic scores summarize millions of alleles and offer a measure of the aggregated influence of a large number of genes with much greater explanatory power than the single genes used by researchers in the past.

Controlling for polygenic scores, in combination with sibling fixed effects models, Conley and colleagues find that birth weight has virtually no effect on any of the midlife outcomes examined. Furthermore, the effect of birth weight does not vary across genetic propensity for each outcome. This double null finding about the main effect of birth weight and its genetic heterogeneity has important implications, because it questions the hypothesis that the effect of in-utero environmental exposures would depend on the genetic vulnerability to the outcome ("genetic heterogeneity"). These findings also suggests that in-utero environmental factors -at least those experienced in "normal times" - do not induce changes in birth weight with long-term influences over the life course. 
The contribution Testing the Only-Child Advantage in Cognitive Development in the Context of China's One-Child Policy by Song and Wang revisits the question about the effect of number of siblings and birth order on children's outcomes, an important question about withinfamily inequality. Prior research has shown that individuals with more siblings have worse educational and economic outcomes and that, among those who have siblings, first-borns have advantages over their younger siblings. Several theories explain these differences: The resource dilution approach argues that more siblings will result in poorer parental investments in each child, driven by a “quality-quantity tradeoff” (Becker and Tomes 1976; Blake 1981). The confluence approach, in turn, suggests that the cognitive environment within the household becomes less rich when there are more younger siblings (Zajonc and Markus 1975). The confluence approach predicts that younger siblings in larger sibships are more disadvantaged compared to only-children and to older siblings, who benefit from teaching younger ones.

In spite of the relevance of this question, capturing the effect of number of siblings is difficult because family size could be correlated with a number of unobserved factors such as personality, ability, social networks, and normative orientations, which could have an independent effect on the outcome of interest. Song and Wang address this question by using the case of China in the context of the one-child policy in force between 1979 and 2015. This policy dramatically increased the number of only-child families regardless of parents' preferences. It provides a unique context to test the effect of being an only child because it induced many families who would have otherwise had more than one child to have only one. The authors cannot claim that having a single child is randomly allocated as it would be in an experiment after all, some of the families with one child under the policy would have had only one child regardless of the policy, and many families did not comply with the policy. The authors therefore 
match children who did and did not receive the "only child policy treatment" based on a number of potential confounders, and compare matched single-children with first-born and later-born children with siblings. Given the pronounced preference for boys over girls in China (Das Gupta et al. 2003) they stratify their analysis by children's gender.

The findings show that only-children have a cognitive advantage only compared with later-born siblings (but not first-born siblings), and only among girls. This result suggests that much of the only-child advantage detected in observational studies might be due to unobserved attributes of only-children and their families. Furthermore, the advantage of only-children over later-born siblings among girls should be interpreted in the context of the strong son preference prevalent in China. Under the one-child policy, girls who are only-children do not have to compete with male siblings for parental investments, while at least some of the later-born girls will have brothers. Given the parental preference for sons, these later-born girls are likely to receive less than their fair share of parental investments and support. In addition to providing strong causal evidence that questions the universality of the only-child advantage, this study highlights the relevance of the cultural and institutional context to understand the consequences of family structure on the wellbeing of the next generation.

The article The Educational Gradient in Health among Children in Immigrant Families by Jackson and Kihara offers a longitudinal assessment of health trajectories of 1 to 15 year-old children born to immigrant and native-born mothers in the United States. The authors ask whether children's early-life health trajectories vary across levels of maternal education, and whether this variation is different for children of immigrants versus children with native-born mothers. 
Jackson and Kihara extend the extant literature in two important ways. By examining educational gradients in health over time rather than at a single point in time they capture trajectories from birth to adolescence. By comparing children born to immigrant and native-born mothers, they account for an important source of heterogeneity in the United States and other wealthy societies with potential consequences for later wellbeing.

A well-established finding in the United States literature is that immigrants have better health outcomes than their native-born co-ethnics, ranging from lower rates of preterm birth to lower mortality in old age. This immigrant health advantage is all the more impressive given that immigrants have, on average, lower levels of education and economic resources than their native counterparts of the same ethnic/racial background. This finding has been called the "immigrant paradox" and has been attributed to the positive selectivity of immigrants, health-enhancing cultural norms about diet and substance use, and stronger social networks of support and assistance (Castañeda et al. 2015; Teruya and Bazargan-Hejazi 2013). The "immigrant paradox" would predict that children of immigrants have better health than native children early in life, but that the immigrant advantage declines over time as children experience "unhealthy acculturation," for instance replacing healthy eating habits with fast food, or losing their closeknit social networks.

Jackson and Kihara's findings question both the "immigrant paradox" and the "unhealthy acculturation" narrative. The authors find that immigrant children have, on average, slightly poorer health than children of native-born mothers, and that health status slightly deteriorates over the early life course among all children, regardless of immigration status. Furthermore, the authors find a pronounced and persistent educational gradient in children's health among both immigrant and native-born families. These findings provide strong evidence questioning the 
perception that being an immigrant provides a silver bullet against poor health, and indicate that education is a paramount resource for both immigrants and native-born children.

Last but not least, Aurino, Schott, Behrman and Penny's contribution, Early-life Nutrition and Adolescent Learning: Gender Parity at Birth, Boys' Advantage by Age 15, examines the association between infants' nutrition status and their cognitive achievement during adolescence in four developing countries -Ethiopia, India, Peru, and Vietnam. This article adds to a growing body of research on the association between nutrition in infancy and individual outcomes later in life (Hoddinott et al. 2013; Nandi et al. 2018). While this literature has focused on outcomes either in early childhood or late adulthood, the authors focus on children's cognitive outcomes during adolescence, a critical stage of the life course characterized by physical and brain maturation and rapid development of skills related to social engagement and emotional control.

The authors examine boys and girls separately, and find that boys have worse nutritional status than girls - measured by length —at age 1, consistent with the literature showing that boys' nutrition in the early years is more vulnerable to food insecurity than girls'. However, boys surpass girls in terms of cognitive ability by age 15 in India and Peru, reach parity with girls in Ethiopia, and lag behind girls only in Vietnam. This finding indicates that boys are able to catch up or even surpass girls from age 1 to age 15 . The authors explain that these patterns of persistence or reversal of the gender gap over the early life course is consistent with gender differences in educational investment and attainment across countries, suggesting the role of families and institutions in altering gender gaps in early achievement. To the extent that these patterns capture causal effects, the findings suggest that supporting nutrition not only during the first year of life but also throughout childhood may have significant payoffs in terms of overall cognitive performance and its parity across genders. 
The five articles included in this volume provide high-quality analyses addressing important issues in the understanding of early-life circumstances, their determinants, and their connection with individual outcomes over the life course in different national settings. We hope this contributions not only increase the scientific understanding of the first years of life but also contribute to decision making oriented to improve the lives of infants, children, and adolescents around the world. 
References

Aizer, A., J. Currie, P. Simon, and P. Vivier. 2017. "Do Low Levels of Blood Lead Reduce Children's Future Test Scores?" American Economic Journal: Applied Economics 10:307341.

Barker, David. 1998. “In Utero Programming of Chronic Disease.” Clinical Science 95:115-28.

Barker, David J. P. 1997. "Maternal Nutrition, Fetal Nutrition, and Disease in Later Life." Nutrition 13(9):807-13.

Barker, David and Clive Osmond. 1986. "Infant Mortality, Childhood Nutrition, and Ischaemic Hearth Disease in England and Wales." The Lancet 1(8489):1077-81.

Bateson, Patrick. 1979. "How Do Sensitive Periods Arise and What Are They For?” Animal Behaviour 27(PART 2):470-86.

Becker, Gary and Nigel Tomes. 1976. "Child Endowments and the Quantity and Quality of Children.” Journal of Political Economy 84(4):S143-62.

Bell, Michelle L. and Keita Ebisu. 2012. "Environmental Inequality in Exposures to Airborne Particulate Matter Components in the United States." Environmental Health Perspectives 120(12):1699-1704.

Ben-Shlomo, Yoav and Diana Kuh. 2002. "A Life Course Approach to Chronic Disease Epidemiology: Conceptual Models, Empirical Challenges and Interdisciplinary Perspectives." International Journal of Epidemiology 31:285-93.

Black, Sandra E., Paul J. Devereux, and Kjell G. Salvanes. 2007. "From the Cradle to the Labor Market? The Effect of Birth Weight on Adult Outcomes." The Quarterly Journal of Economics 122(1):409-39.

Blake, Judith. 1981. "Family Size and the Quality of Children.” Demography 18(4):421-42.

Brown, Robert. 2005. “Critical Period.” Pp. 325-27 in Encyclopedia of Human Development. Sage.

Castañeda, Heide, Seth M. Holmes, Daniel S. Madrigal, Maria-Elena DeTrinidad Young, Naomi Beyeler, and James Quesada. 2015. "Immigration as a Social Determinant of Health." Annual Review of Public Health 36:375-92.

Caudillo, Monica and Florencia Torche. 2014. "Exposure to Local Homicides and Early Educational Achievement in Mexico." Sociology of Education 87(2):89-105.

Cunha, Flavio and James Heckman. 2007. "The Technology of Skill Formation.” American Economic Review, Papers and Proceedings 97(2):31-47.

Evans, Gary W., Dongping Li, and Sara Sepanski Whipple. 2013. "Cumulative Risk and Child Development." Psychological Bulletin 139(6):1342-96.

Farah, Martha J., David M. Shera, Jessica H. Savage, Laura Betancourt, Joan M. Giannetta, Nancy L. Brodsky, Elsa K. Malmud, and Hallam Hurt. 2006. "Childhood Poverty : Specific Associations with Neurocognitive Development." Brain Research 1110:166-74.

Forsdahl, Anders. 1977. "Are Poor Living Conditions in Childhood and Adolescence an Important Risk Factor for Arteriosclerotic Heart Disease?" British Journal of Preventive 
and Social Medicine 31:91-95.

Forsdahl, Anders. 1978. "Living Conditions in Childhood and Subsequent Development of Risk Factors for Arteriosclerotic Heart Disease." Journal of Epidemiology and Community Health 32:34-37.

Geronimus, Arline T. 1992. "The Weathering Hypothesis and the Health of African-American Women and Infants: Evidence and Speculations." Ethnicity \& Disease 2:207-21.

Gluckman, Peter, Mark Hanson, Cyrus Cooper, and Kent Thornburg. 2008. "Effects of In-Utero and Early-Life Conditions on Adult Health and Disease." New England Journal of Medicine 359(1):61-73.

Godfrey, Keith M. and David J. P. Barker. 2001. "Fetal Programming and Adult Health.” 4.

Grossman, Daniel and David Slusky. 2017. The Effect of an Increase in Lead in the Water System on Fertility and Birth Outcomes: The Case of Flint, Michigan. Working Paper http://www2.ku.edu/ kuwpaper/2017Papers/201703.pdf.

Das Gupta, Monica, Zhenghua Jiang, Bohua Li, Zhenming Xie, Chung Woojin, and Hwa Ok Bae. 2003. "Why Is Son Preference so Persistent in East and South Asia? A Cross-Country Study of China, India and the Republic of Korea." Journal of Development Studies 40(2):153-87.

Hair, Nicole L., Jamie L. Hanson, Barbara L. Wolfe, and Seth D. Pollak. 2015. "Association of Child Poverty, Brain Development, and Academic Achievement." JAMA Pediatrics 169(9):822-29.

Harrell, Erika, Lynn Langton, Marcus Berzofsky, Lance Couzens, and Hope Smiley-Mcdonald. 2014. Household Poverty and Nonfatal Violent Victimization, 2008-2012.

Hartshorne, Joshua K., Joshua B. Tenenbaum, and Steven Pinker. 2018. "A Critical Period for Second Language Acquisition : Evidence from 2 / 3 Million English Speakers." Cognition 177:263-77.

Hertzman, Clyde and Tom Boyce. 2010. "How Experience Gets Under the Skin to Create Gradients in Developmental Health.” Annual Review of Public Health 31:329-47.

Hobel, Calvin J. 2004. "Stress and Preterm Birth.” Clinical Obstetrics and Gynecology 47(4):856-80.

Hoddinott, John, Jere R. Behrman, John A. Maluccio, Paul Melgar, Agnes R. Quisumbing, Manuel Ramirez-Zea, Aryeh D. Stein, Kathryn M. Yount, and Reynaldo Martorell. 2013. "Adult Consequences of Growth Failure in Early Childhood." American Journal of Clinical Nutrition 98(5):1170-78.

Hoynes, Hilary, Marianne Page, and Ann Huff Stevens. 2011. "Can Targeted Transfers Improve Birth Outcomes?. Evidence from the Introduction of the WIC Program." Journal of Public Economics 95(7-8):813-27.

Hoynes, Hilary W., Douglas L. Miller, and David Simon. 2015. "Income, the Earned Income Tax Credit, and Infant Health.” American Economic Journal: Economic Policy 7(1):172211.

Johnson, Sara B., Jenna L. Riis, and Kimberly G. Noble. 2016. "State of the Art Review: Poverty 
and the Developing Brain.” Pediatrics 137(4):1-18.

Kermack, W. O., A. G. McKendrick, and P. L. McKinlay. 1934. "Death-Rates in Great Britain and Sweden. Some General Regularities and Their Significance.” Lancet 226:698-703.

King, Katherine E., Jeffrey D. Morenoff, and James S. House. 2011. "Neighborhood Context and Social Disparities in Cumulative Biological Risk Factors.” Psychosomatic Medicine 73(7):572-79.

Knudsen, Eric I. 2004. "Sensitive Periods in the Development of the Brain and Behavior," Journal of Cognitive Neuroscience, 16(8): 1412-1425, 2004." Journal of Cognitive Neuroscience 16(8):1412-25.

Kuh, Diana and George Davey Smith. 1993. "When Is Mortality Risk Determined? Historical Insights into a Current Debate." Social History of Medicine 6(1):101-23.

Lenneberg, E. 1967. Biological Foundations of Language. New York: Wiley.

Lynch, John and George Davey Smith. 2005. "A Life Course Approach to Chronic Disease Epidemiology." Annual Review of Public Health 26:1-35.

McEwen, Bruce S. and Eliot Stellar. 1993. "Stress and the Individual: Mechanisms Leading to Disease.” Archives of Internal Medicine 153:2093-2101.

McEwen, Craig A. and Bruce S. McEwen. 2017. "Social Structure, Adversity, Toxic Stress, and Intergenerational Poverty: An Early Childhood Model.” Annual Review of Sociology 43:445-72.

McLean, Mark, Andrew Bisits, Joanne Davies, Russell Woods, Philip Lowry, and Roger Smith. 1995. "A Placental Clock Controlling the Length of Human Pregnancy." Nature Medicine 1(5):460-63.

Michigan Department of Health and Human Services. 2017. Flint Perinatal Metrics Analyses. https://www.michigan.gov/documents/mdhhs/Flint_Perinatal_Metrics_Updated_10.4.17_60 3174_7.pdf.

Muller, Christopher, Robert J. Sampson, and Alix S. Winter. 2018. "Environmental Inequality: The Social Causes and Consequences of Lead Exposure." Annual Review of Sociology 44(1):263-82.

Nandi, Arindam, Jere R. Behrman, Sanjay Kinra, and Ramanan Laxminarayan. 2018. "EarlyLife Nutrition Is Associated Positively with Schooling and Labor Market Outcomes and Negatively with Marriage Rates at Age 20-25 Years: Evidence from the Andhra Pradesh Children and Parents Study (APCAPS) in India." Journal of Nutrition 148(1):140-46.

Noble, Kimberly G., Suzanne M. Houston, Natalie H. Brito, Elizabeth R. Sowell, and Et Al. 2015. "Family Income, Parental Education and Brain Structure in Children and Adolescents." Nature Neuroscience 18(5):773-78.

Oreopoulos, Philip, Mark Stabile, Randy Walld, and Leslie L. Roos. 2012. "Short-, Medium-, and Long-Term Consequences of Poor Infant Health: An Analysis Using Siblings and Twins." Journal of Human Resources 43(1):88-138.

Rice, Deborah and Stan Barone. 2000. "Critical Periods of Vulnerability for the Developing Nervous System: Evidence from Humans and Animal Models.” Environmental Health 
Perspectives 108(S3):511-33.

Rutter, Michael. 1989. "Pathways from Childhood to Adult Life." Journal of Child Psychology and Psychiatry 30(1):23-51.

Sharkey, Patrick. 2010. "The Acute Effect of Local Homicides on Children's Cognitive Performance." PNAS 107(26):11733-38.

Shonkoff, Jack P. and Deborah A. Phillips. 2000. From Neurons to Neighborhoods: The Science of Early Childhood Development. National Academies Press.

Teruya, Stacey and Shahrzad Bazargan-Hejazi. 2013. "The Immigrant and Hispanic Paradoxes: A Systematic Review of Their Predictions and Effects." Hispanic Journal of Behavioral Science 35(4):486-509.

Torche, Florencia. 2015. "Intergenerational Mobility and Equality of Opportunity." European Journal of Sociology 56(03):343-71.

Torche, Florencia. 2018. "Prenatal Exposure to an Acute Stressor and Children's Cognitive Outcomes." Demography 55:1611-39.

Torche, Florencia and Dalton Conley. 2015. "A Pound of Flesh : The Use of Birthweight as a Measure of Human Capital Endowment in Economics." Oxford Handbooks Online https://www.oxfordhandbooks.com/view/10.1093/oxfor.

Torche, Florencia and Ghislaine Echevarría. 2011. "The Effect of Birthweight on Childhood Cognitive Development in a Middle-Income Country." International Journal of Epidemiology 40(4):1008-18.

Wodtke, Geoffrey T., Felix Elwert, and David J. Harding. 2016. "Neighborhood Effect Heterogeneity by Family Income and Developmental Period." American Journal of Sociology 121(4):1168-1222.

Zajonc, R. B. and Gregory B. Markus. 1975. "Birth Order and Intellectual Development." Psychological Review 82(1):74-88. 